\title{
Facebook au service d'une éducation au changement climatique en contexte de famille
}

Michel T. Léger et Shawn Martin

\section{(2) OpenEdition}

Édition électronique

URL : http://journals.openedition.org/ere/5892

DOI : $10.4000 /$ ere. 5892

ISSN : 2561-2271

Éditeur

Centr'ERE

Référence électronique

Michel T. Léger et Shawn Martin, «Facebook au service d'une éducation au changement climatique en contexte de famille », Éducation relative à l'environnement [En ligne], Volume 15 - 2 | 2020, mis en ligne le 15 novembre 2020, consulté le 25 février 2021. URL : http://journals.openedition.org/ere/5892 ; DOI : https://doi.org/10.4000/ere.5892

Ce document a été généré automatiquement le 25 février 2021. 


\title{
Facebook au service d'une éducation au changement climatique en contexte de famille
}

\author{
Michel T. Léger et Shawn Martin
}

Ce projet n'aurait pas été possible sans l'appui financier du Conseil de recherche en sciences humaines du Canada (Subvention de développement Savoir, 2015). Il nous importe aussi de reconnaitre la contribution de l'analyste Robert Robichaud, consultant pour Énergie NouveauBrunswick, et celle de Shawn Martin, étudiant à la Maitrise en environnement de l'Université de Moncton et principal assistant de recherche pour cette étude.

1 Au Canada, comme c'est le cas dans plusieurs autres pays, il semble que les citoyennes et citoyens montrent de plus en plus d'intérêt pour l'environnement et un désir croissant d'agir de façon plus responsable envers leur milieu de vie, sans toutefois réussir à concrétiser cette intention en actions susceptibles de contribuer à la résolution des problèmes environnementaux (Whitmarsh et O'Neill, 2011). Cherchant à mieux définir l'action environnementale, Marleau (2009) cite des auteurs comme Emmons (1997), Clover (2002) et Garcia (2004) pour suggérer que la décision d'agir de manière écoresponsable est fondée sur certaines compétences en matière de résolution de problèmes comme l'analyse et la planification. Marleau (2009) ajoute que le choix d'adopter une action écologique demande un niveau de conscience environnementale de base ainsi qu'une intention de vouloir préserver l'environnement. Or comment pallier l'écart entre une conscience environnementale et l'action écologique concrète, tel qu'observé par Kollmuss et Agyeman (2002)?

2 À partir de nos travaux antérieurs sur le développement de compétences environnementales (Léger et coll., 2014 ; Pruneau et coll., 2015 ; Pruneau et coll., 2013), nous comprenons mieux les processus qui sous-tendent le développement de compétences comme la pensée prospective, l'analyse du risque en environnement et la résolution de problèmes environnementaux. Nous comprenons aussi mieux le rôle de ces compétences pour la mise en œuvre d'actions environnementales, surtout en contexte de famille (Léger et Pruneau, 2014, 2013, 2012). Plus récemment, nos travaux 
sur les compétences numériques (Léger et Freiman, 2016, 2015 ; Freiman et coll., 2017) ont montré plusieurs similarités entre les compétences nécessaires à l'adoption d'actions environnementales et celles associées au développement d'une meilleure littératie numérique. Nous nous sommes donc demandé si l'utilisation de technologies de l'information et de la communication (TIC) pouvaient contribuer à combler l'écart entre l'intention d'agir de façon écologique et l'action écoresponsable concrète, ou ce que Jensen et Schnack (1997) appellent la « compétence d'action » environnementale.

\section{Les technologies et l'action environnementale}

Tout comme les enjeux environnementaux, les technologies occupent une place importante au $21^{\mathrm{e}}$ siècle. Plusieurs pays cherchent actuellement à aider leurs citoyennes et citoyens à se trouver une place dans l'économie numérique en implantant divers programmes qui visent à faciliter le développement de compétences numériques. Au Canada, la plus récente initiative du gouvernement fédéral en matière de littératie numérique s'intitule Canada numérique 150 (Industrie Canada, 2014) ; il s'agit d'accentuer les initiatives permettant aux Canadiennes et aux Canadiens de prendre pleinement avantage des possibilités transformationnelles offertes à l'ère du numérique. En fait, d'après des organismes de compilation de données Web comme Internet World Stats ( www.internetworldstats.com) et We Are Social (www.wearesocial.com), environ $50 \%$ de la population mondiale utilisait Internet en 2017. Tout aussi impressionnant est le nombre d'individus qui utilisent régulièrement les médias sociaux, estimé par ces mêmes sources à environ 2,8 milliards, ce qui représente $37 \%$ de la population mondiale.

4 Une recension des écrits sur le rôle potentiel des TIC et autres technologies sur le choix de se comporter de façon plus écoresponsable n'a permis de repérer que très peu d'articles scientifiques. La plupart des quelques études publiées parlent d'une influence surtout positive des plateformes de réseautage social, comme Facebook et Twitter, sur l'adoption de modes de vie plus écologiques. Par exemple, Bell et coll. (2016) rapportent que les médias sociaux peuvent contribuer à un plus haut niveau de conscience environnementale chez les adolescents d'une famille, ainsi qu'une réduction en termes de consommation d'énergie à la maison. Ces auteurs notent aussi que les médias sociaux peuvent servir d'appui à l'adoption d'actions écologiques chez une personne qui est déjà prête à changer d'après le Modèle de changement comportemental transthéorique de Prochaska et coll. (1992), c'est-à-dire lorsqu'une personne a déjà l'intention d'agir dans un avenir immédiat. Dans une autre étude, Sweeney et coll. (2014) ont montré que les médias sociaux peuvent mener à un sentiment d'autodétermination chez une personne qui essaie d'intégrer des habitudes proenvironnementales.

5 Notre étude porte un regard novateur sur l'écocitoyenneté en croisant les cadres théoriques de la compétence environnementale, de l'action environnementale et de la littératie numérique. Nous nous demandons si le choix d'intégrer des modes de vie plus écologiques à la maison pouvait être positivement influencé par un fil d'actualité Facebook qui comporte des publications régulières sur des enjeux environnementaux ainsi que des conseils en matière d'écocitoyenneté. En d'autres mots, un tel renforcement virtuel serait-il de nature à stimuler le développement et le maintien d'actions environnementales concrètes? 


\section{La famille comme contexte de transformation socioécologique}

6 Presque toute culture considère la famille comme unité d'organisation sociale de base. Statistiques Canada (2017) définit la «famille recension » comme une personne ou un groupe de personnes qui «vivent dans un même endroit». Pour Le Bourdais et coll. (1991), cette définition est trop unidimensionnelle puisqu'elle met l'accent sur la place plutôt que sur les relations interpersonnelles et les dynamiques sociales qui font partie intégrale de la vie familiale. D'autres auteurs dans les domaines de la sociologie et de la psychologie comportementale (Widmer et coll., 2004; Claffey et Mickelson, 2009; Neilson et Stanfors, 2014) ont souligné la place importante des interactions sociales dans la famille équilibrée sur le plan fonctionnel. Aujourd'hui, dans un monde de plus en plus numérique, de telles interactions intrafamiliales sont soumises à l'influence de la technologie, comme tout autre aspect de la vie. Notamment, les différentes plateformes de médias sociaux ont significativement transformé la façon que nous communiquons les uns avec les autres. Étant donné la place de la famille comme dénominateur commun de la structure sociétale partout au monde, étant donné aussi l'influence importante des médias sociaux virtuels comme Facebook et Twitter sur les interactions dans et au-delà de la famille, nous considérons que la famille est un contexte de transformation socioécologique sur lequel il importe de se pencher.

7 Bien qu'en nombre limité, il existe quelques études qui examinent l'adoption d'actions écoresponsables en contexte de famille. En Australie, le Queensland Youth Environmental Council (2009) rapporte que faire partie d'une famille pro-environnementale constitue un facteur important d'action environnementale chez les jeunes âgés de 12 à 24 ans. Pour sa part, Payne $(2005,2010)$ a obtenu des résultats semblables, ajoutant que les enfants imitent souvent les pratiques écologiques de leurs parents. Dans une étude internationale menée par l'Organisation de coopération et de développement économique (OCDE, 2008), on rapporte qu'un membre d'une famille peut, en fait, influencer positivement un autre membre à adopter une action environnementale comme le recyclage. Au Canada, nos propres recherches sur l'écocitoyenneté en contexte de famille soulignent l'importance de développer des valeurs familiales écocentriques et des compétences environnementales collectives, comme la pensée prospective, chez une famille qui cherche à adopter des modes de vie écologiques à la maison (Léger et Pruneau, 2014, 2013, 2012).

\section{Objectifs de recherche}

8 Notre étude vise à combler un manque de recherches portant sur le développement d'une écocitoyenneté familiale à l'aide de technologies numériques. Plus spécifiquement, comme contribution au champ de l'éducation relative à l'environnement en contexte informel (Sauvé, 1997), nous voulons mieux comprendre le rôle potentiel du réseau social Facebook dans les efforts de réduction de consommation en électricité chez une famille qui souhaite adopter des modes de vie plus écologiques. À cet effet, nous avons créé une page Facebook sur la question de l'économie d'énergie et invité des familles à s'y joindre comme " amies », formant ainsi un groupe privé. La question de recherche suivante a guidé notre investigation: 
Comment le réseau social Facebook peut-il influencer l'adoption d'actions permettant de contribuer à la mitigation des changements climatiques en contexte de familles qui vivent en milieu urbain? À partir de cette question, trois objectifs de recherche spécifiques sont formulés :

- Décrire le fréquentation et l'utilisation d'une page Facebook portant sur l'économie d'énergie par des familles vivant dans petit centre urbain.

- Mesurer l'impact de ce média sur la consommation mensuelle d'électricité par les familles participantes.

- Comprendre l'expérience vécue par des familles qui tentent d'adopter des actions proenvironnementales en participant à une communauté virtuelle.

\section{Méthodologie}

9 Cette étude relève d'un paradigme de recherche surtout qualitatif, bien que nous avons aussi eu recours à l'analyse descriptive de données quantitatives pendant l'expérimentation. Comme structure méthodologique, nous avons choisi l'étude de cas multiples (Stake, 1995), où chaque cas représentait une famille qui cherchait à adopter des modes de vie plus écologiques. Les familles participantes, provenant surtout de Dieppe, une petite ville au sud-est du Nouveau-Brunswick, ont été sélectionnées par échantillonnage théorique (Creswell, 2007) d'après les trois critères d'inclusion suivants: elles devaient avoir manifesté une intention de vivre de façon plus écoresponsable comme collectivité ; elles devaient avoir accès à Internet à la maison et savoir se servir d'un ordinateur ou d'un téléphone intelligent; les membres de la famille devaient être actifs sur le réseau social Facebook.

Avec la collaboration du District scolaire francophone Sud, nous avons envoyé plus de 150 courriels d'invitation à la recherche aux parents d'enfants âgés de 13 à 17 ans de la région de Dieppe. En tout, 22 familles ont répondu à l'appel en demandant plus d'information. De ces répondants, 15 familles ont accepté de participer à notre étude et, après application des critères d'inclusion, 12 familles au total ont finalement été retenues. Sur les quatre mois d'expérimentation, ce groupe de familles participantes ont reçu des informations et des conseils au sujet des changements climatiques de la part d'une "famille modèle ", choisie par le chercheur, qui mène déjà un style de vie familiale écoresponsable. Dans un groupe privé Facebook duquel faisaient partie les 12 familles de l'étude, la "famille modèle » affichait régulièrement des publications conçues pour stimuler l'activité de partage au sein du groupe privé. Ces publications visaient à informer les membres du groupe sur les enjeux environnementaux de la surconsommation d'énergie à la maison, et à leur offrir des conseils pour mener une vie de famille plus écoresponsable en général. L'idée était d'établir un réseau de partage social via Facebook afin de voir si l'expérience virtuelle contribuerait à l'adoption d'actions moins énergivores dans la famille.

11 Sur le plan opérationnel, la recherche comportait deux types de données. Dans un premier temps, nous avons compilé des statistiques descriptives à partir de données quantitatives issues de notre examen des activités Facebook sur la période d'expérimentation. Chaque jour pendant quatre mois, nous avons comptabilisé chaque publication, chaque commentaire et chaque "j'aime» de la part des familles participantes. Ceci nous a permis de mieux connaitre le type d'usage que faisaient les familles participantes de l'outil Facebook. À l'aide de tableaux de fréquences et de 
graphiques, nous avons pu analyser les activités Facebook de l'ensemble des familles du groupe privé. De plus, la quantité mensuelle de kilowattheures $(\mathrm{kWh})$ utilisés a été notée pour les mois de l'étude, et comparée avec celle utilisée pour les mêmes mois de l'année précédente. Ces données correspondent à des valeurs objectives provenant de la lecture des compteurs d'électricité d'Énergie NB.

Pour ce qui est de l'aspect qualitatif de notre étude, correspondant à la dimension méthodologique principale de la recherche, nous avons mené des entretiens semistructurés avec chacune des familles participantes à la fin du projet. Ces entretiens ont été enregistrés et ensuite transcrits Ils ont fait l'objet d'une analyse de contenu thématique telle que décrite par Paillé et Mucchielli (2012). À partir de cette stratégie analytique, nous avons identifié, organisé et décrit un ensemble de catégories phénoménologiques (ou thèmes) autour du discours de chaque famille participante. Durant le processus de l'analyse, deux chercheurs ont traité de façon indépendante le corpus de données qualitatives, lui apposant des codes initiaux pour représenter les thèmes émergents tels qu'ils les voyaient. Ces deux chercheurs ont alors comparé leurs résultats relatifs à chaque cas dans un processus corroboratif d'analyse thématique, ajoutant ainsi à la validité des thèmes retenus. La validité interne des résultats a aussi été assurée par le partage des transcriptions d'entretiens avec les familles afin qu'elles puissent corroborer, à leur tour, la fidélité des rapports écrits avant que ceux-ci soient soumis à l'analyse thématique. Au bilan, nous avons repéré les similarités et les différences entre les cas étudiés pour enfin produire un bilan des principaux thèmes communs, nous donnant ainsi un portrait plus global de l'expérience de transformation socioécologique en famille avec Facebook.

Avant et pendant l'étude, la confidentialité a été assurée dans tous les aspects de la recherche. À cet effet, nous avons assigné des pseudonymes à tous les participants et nous avons gardé toutes les données sous clé, les rendant disponibles seulement aux chercheurs impliqués. Le consentement libre et éclairé de chaque participant a aussi été assuré par la signature d'un formulaire à cet effet distribué aux personnes concernées avant de démarrer l'étude. Enfin, l'approbation éthique pour cette étude a été obtenue par le Comité d'éthique de la recherche avec les êtres humains de l'Université de Moncton, l'institution d'attache des chercheurs.

\section{Résultats}

14 À partir des données quantitatives sur l'utilisation de Facebook enregistrées quotidiennement sur les quatre mois de l'expérimentation, nous avons pu établir certains patterns d'activité. Nous avons caractérisé le niveau d'activité des familles participantes à partir du nombre de «j'aime », de publications libres et du nombre de commentaires faits en réponse aux publications d'autres familles du réseau. Ainsi, une famille participante avec un total de 1 à 5 traces d'activité («j'aime»; publication ; commentaire) a été catégorisée comme "peu active ». Une famille considérée " active " sur le groupe privé Facebook comptait entre 6 à 20 traces d'activité, tandis qu'une famille «très active » en comptait plus de 20 . En terme d'économie d'électricité sur la période d'expérimentation, nous avons noté que plus de la moitié des familles participantes (7/12) ont réussi de telles économies à la maison, affichant une réduction moyenne de $10 \%$ sur l'ensemble des quatre mois. Ceci représente une réduction d'en moyenne $306 \mathrm{kWh}$ par famille participante sur quatre mois, une valeur qui se traduit 
par $987 \mathrm{~kg}$ de $\mathrm{CO}_{2}$ (ou environ 1 tonne métrique de $\mathrm{CO}_{2}$ ) non libérés dans l'atmosphère. Le Tableau 1 résume les résultats quantitatifs des familles participantes, tandis que les Figures 1 et 2 illustrent plus spécifiquement leur activité sur Facebook en termes de fréquence de leurs commentaires et du nombre de leurs publications. Un constat intéressant par rapport aux deux graphiques présentés est la différence marquée des commentaires et des publications laissés dans la première moitié de l'étude, en comparaison à ceux laissés dans les deux derniers mois de l'expérimentation.

Tableau 1 : Participation des familles du groupe privé Facebook

\begin{tabular}{|c|c|c|c|c|}
\hline \multicolumn{2}{|c|}{$\begin{array}{c}\text { Famille } \\
\text { participante }\end{array}$} & $\begin{array}{c}\text { Niveau d'activité } \\
\text { Facebook }\end{array}$ & $\begin{array}{c}\text { Moyenne de } \\
\text { kWh économisés }\end{array}$ & $\begin{array}{c}\text { Total de } \mathrm{kg}_{\text {de }} \mathrm{CO}_{2} \\
\text { non libéré }\end{array}$ \\
\hline 1 & Bernier & Peu active & 0 & 0 \\
\hline 2 & Pelletier & Très active & 335 & 84 \\
\hline 3 & Fournier & Peu active & 0 & 0 \\
\hline 4 & Giroux & Très active & 587 & 148 \\
\hline 5 & Jones & Très active & 633 & 70 \\
\hline 6 & Allard & Active & 276 & 0 \\
\hline 7 & Belliveau & Très active & 0 & 155 \\
\hline 8 & Dallaire & Très active & 587 & 229 \\
\hline 9 & Roberts & Très active & 910 & 0 \\
\hline 10 & Thériault & Peu active & 0 & 87 \\
\hline 11 & Maillet & Active & 340 & 0 \\
\hline 12 & Chouinard & Peu active & 0 & $\Sigma=987 \mathrm{~kg}$ de $\mathrm{CO}_{2}$ \\
\hline
\end{tabular}

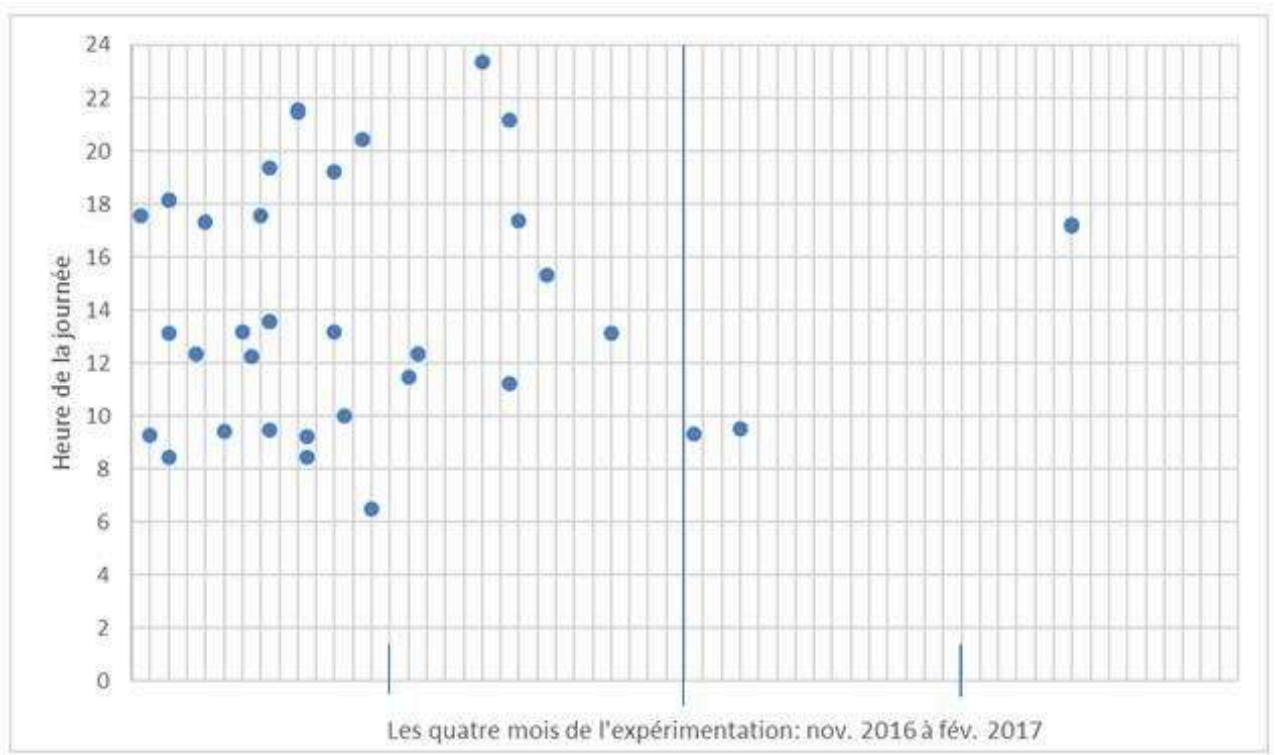


Figure 1. Nombre de commentaires partagés par les familles du groupe privé Facebook

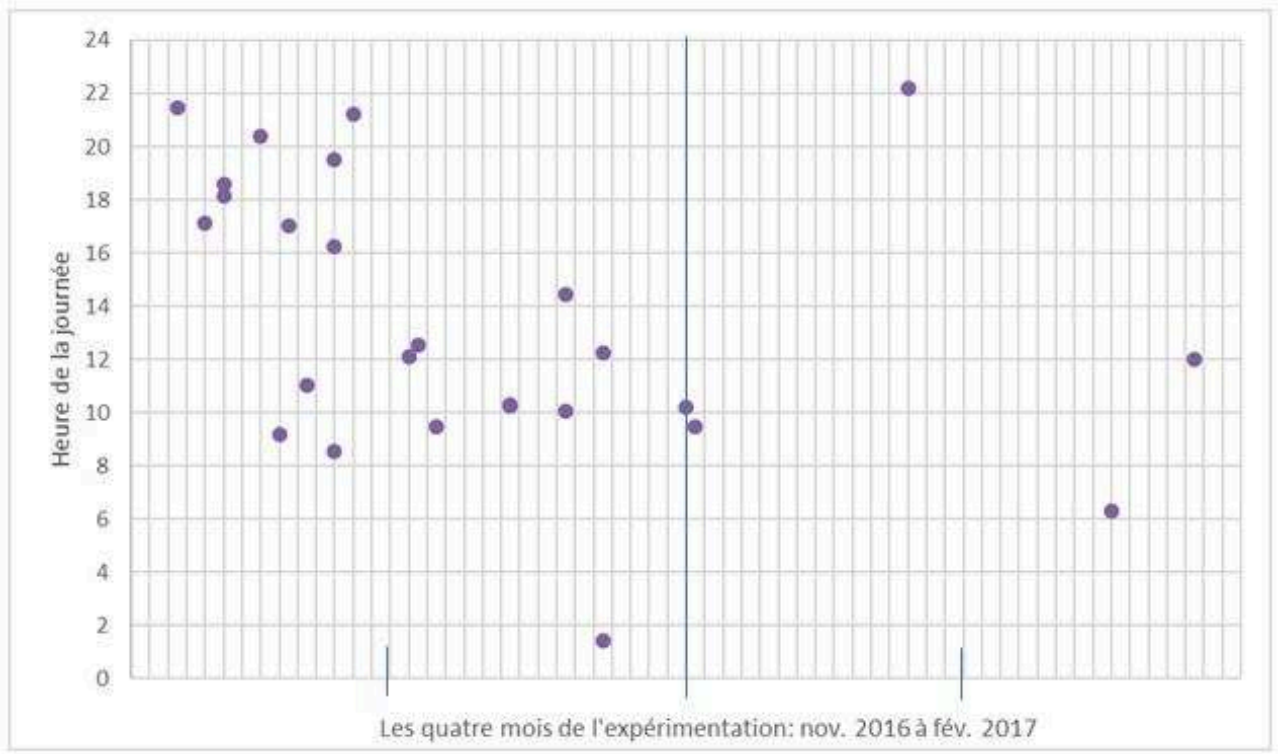

Figure 2. Nombre de publications partagées par les familles du groupe privé Facebook groupe Facebook par les familles participantes pendant la période d'expérimentation. Or, ils ne permettent pas en eux-mêmes de comprendre l'expérience de transformation socioécologique telle que vécue dans chacune des familles ayant participé au réseau social virtuel que nous avons mis en place. Pour répondre à cet objectif, nous avons eu recours à des entretiens de fin de projet avec chacune des familles participantes. Voici quelques questions posées à ces entretiens: Quelles actions avez-vous tenté d'adopter comme famille pendant le projet? Décrivez votre expérience de changements comme famille à l'aide de Facebook. Que pensez-vous de l'appui reçu par la "famille modèle»? Comment votre expérience de changement a-t-elle été influencée par vos interactions avec les membres $d u$ groupe privé Facebook? Quels défis votre famille a-t-elle rencontrés au cours du projet?

Après une analyse de contenu thématique des données qualitatives (Paillé et Mucchielli, 2012), nous avons repéré un certain nombre de thèmes émergents qui semblent corroborer le succès des actions tel que montré par les résultats quantitatifs. Dans le Tableau 2, nous présentons ces thèmes qualitatifs, ainsi que des éléments du corpus d'entrevues à titre d'exemples de données ayant mené au codage initial. 
Tableau 2 : Résultats de l'analyse de contenu thématique

\begin{tabular}{|l|l|}
\hline \multicolumn{1}{|c|}{ Thèmes majeurs } & \multicolumn{1}{|c|}{ Exemples d'éléments du corpus d'entrevues } \\
\hline $\begin{array}{l}\text { Actions choisies qui sont } \\
\text { relativement simples }\end{array}$ & $\begin{array}{l}\text { - Réduction de l'eau chaude consommée (douche, vaisselle) } \\
\text { - Réduction de la température ambiante (thermostat programmé) }\end{array}$ \\
\hline $\begin{array}{l}\text { Difficulté de changer les } \\
\text { habitudes. }\end{array}$ & $\begin{array}{l}\text { - Le changement prend du temps. } \\
\text { - C'est difficile de sacrifier des éléments de confort. }\end{array}$ \\
\hline $\begin{array}{l}\text { Conscience environnementale } \\
\text { plus élevée }\end{array}$ & $\begin{array}{l}\text { - Les familles sont plus conscientes des problèmes } \\
\text { environnementaux. } \\
\text { - Les familles sont plus conscientes de leur impact sur } \\
\text { l'environnement. }\end{array}$ \\
\hline $\begin{array}{l}\text { Engagement familial } \\
\text { plus élevé }\end{array}$ & $\begin{array}{l}\text { - Tous les membres de la famille sont impliqués dans le } \\
\text { changement. } \\
\text { - Les participants sont encouragés par l'appui des autres familles }\end{array}$ \\
\hline $\begin{array}{l}\text { Participation plus élevée des } \\
\text { enfants de la famille }\end{array}$ & $\begin{array}{l}\text { - Les enfants font des rappels d'agir auprès de leurs parents. } \\
\text { - Les enfants font plusieurs actions simples (éteindre les } \\
\text { lumières). }\end{array}$ \\
\hline $\begin{array}{l}\text { Plus de conversations sur } \\
\text { l'environnement en famille }\end{array}$ & $\begin{array}{l}\text { - Les familles discutent de valeurs environnementales. } \\
\text { - Les familles discutent des problèmes environnementaux. }\end{array}$ \\
\hline
\end{tabular}

\section{Discussion et conclusions}

17 Aujourd'hui, les gens de partout au monde sont préoccupés par des problèmes environnementaux de plus en plus sévères comme la désertification des terres arables, la pollution atmosphérique et, bien sûr, les changements climatiques. Au niveau sociétal, on est à la recherche de solutions. Des initiatives à grande échelle comme l'élimination de notre dépendance systémique aux carburants fossiles seront incontournables si on veut atteindre les objectifs établis par l'Accord de Paris, dont celui de limiter l'augmentation de la température moyenne globale à un maximum de 2 degrés Celsius (CCNUCC, 2015). Par contre, à notre avis, il est quand même important de valoriser les changements à plus petite échelle, notamment le développement d'un engagement envers l'environnement comme celui qu'ont manifesté les familles de notre étude dans leurs propres efforts d'écocitoyenneté locale. À l'ère numérique, il est particulièrement pertinent, selon nous, d'inclure les technologies de communication dans la lutte sociétale contre les changements climatiques. Certes, ces technologies ne constituent pas à elles seule la solution aux grands problèmes environnementaux, d'autant plus qu'elles contribuent en quelque sorte à certains de ces problèmes en raison, par exemple, de l'exploitation des ressources nécessaires à la fabrication des appareils et de la pollution liée à leur surproduction et au stockage des données. Or des recherches comme celle-ci montrent les technologies de l'information et de la communication (TIC) comme celles qui sont à la base des médias sociaux numériques, peuvent servir à développer des actions écoresponsables collectives, en contexte de famille entre autres.

Bien que notre devis méthodologique ne permette pas d'attribuer directement les économies d'électricité de nos familles participantes à leur utilisation de TIC comme Facebook, nos résultats tendent à montrer une relation possible, justifiant à notre avis plus de recherches sur la place des technologies numériques dans l'intégration de modes de vie plus écologiques en contexte de famille. En effet, selon nos résultats, des actions proenvironnementales concrètes ont été adoptées par la plupart des familles utilisatrices d'un site Facebook destiné à cet effet. Il est à noter cependant que ces 
actions étaient simples : par exemple, réduire la température ambiante dans la maison, limiter l'utilisation d'eau chaude ou éteindre les lumières en quittant une pièce de la maison.

Quant aux les thèmes qui ont émergé de notre étude, notons que les familles ont trouvé qu'il était difficile de se défaire de leurs habitudes énergivores à la maison. En effet, comme le suggère Maiteney (2002), il n'est pas facile de changer des comportements de confort pour des modes de vie plus écoresponsables. Malgré ce défi, toutes les familles ont indiqué que leur participation au groupe Facebook a mené à un plus haut degré de conscientisation environnementale collective. Les participants ont aussi signalé que les enfants ont joué un rôle actif dans les efforts collectifs de réduction d'électricité, rappelant régulièrement à leurs parents de maintenir les actions familiales visées. En plus des rappels reçus par leurs enfants, les parents ont rapporté être encouragés par le support ressenti de la part des autres familles du groupe Facebook. Les familles ont aussi précisé que leur participation à ce groupe a suscité davantage de conversations familiales au sujet de l'environnement et soulevé des discussions sur les valeurs familiales.

En conclusion, selon nos résultats, il semble que l'utilisation du réseau social Facebook peut stimuler l'adoption collective d'actions qui réduisent la consommation d'électricité à la maison. Participer à un groupe privé Facebook semble aussi avoir contribué à augmenter le niveau familial de conscience environnementale et d'engagement environnemental. Ceci est conforme aux conclusions d'autres auteurs comme Bell et coll. (2016). Enfin, bien que ces résultats soient positifs, ils sont issus d'une étude exploratoire et ainsi, ils ne sont pas généralisables. Selon nous, des recherches subséquentes sur le rôle des médias sociaux pour le développement d'actions collectives écocitoyennes sont donc nécessaires. Plusieurs questions demeurent. Par exemple, est-ce que des plateformes de réseautage social technologique autre que Facebook, comme Twitter, Instagram ou le simple courrier électronique, pour en nommer que trois, peuvent aussi avoir une incidence sur la réduction de consommation énergétique chez la famille? Quels sont les limites des technologies en général sur le développement d'un sentiment de pouvoir agir environnemental, non seulement en contexte collectif, comme dans la famille, mais aussi chez l'individu?

\section{BIBLIOGRAPHIE}

Bell, B. T., Toth, N., Little, L., et Smith, M. A. (2016). Planning to Save the Planet : Using an Online Intervention Based on Implementation Intentions to Change Adolescent Self-Reported EnergySaving Behavior. Environment and Behavior, 48(8), 1049-1072.

Bristow, A., Pridmore, A., Tight, M., May, T., Berkout, F. et Harris, M. (2004). How can we reduce carbon emissions from transport? Tyndall Center Technical Report. www.tyndall.ac.uk/research/ theme 2/final_report/it1_7.pdf

Claffey, T. S. et Mickelson, D. (2009). Division of Household Labor and Distress : The Role of Perceived Fairness for Employed Mothers, Sex roles, 60, 11-12. 
Clover, D. (2002). Traversing the gap : concientización, educative-activism in environmental adult education. Environmental Education Research, 8(3), p. 315-323.

Convention-cadre des Nations unies sur les changements climatiques (CCNUCC) (2015). Accord de Paris. https://unfccc.int/files/essential_background/convention/application/pdf/

french_paris_agreement.pdf

Creswell, J. W. (2007). Qualitative inquiry \& research design : Choosing among five approaches.

Thousand Oaks, CA : Sage.

Emmons, K. M. (1997). Perspective on environmental action : Reflection and revision through practical experience. The Journal of Environmental Education, 29(1), p. 34-44.

Garcia, J. E. (2004). Educación ambiental, constructivismo y complejidad. Sevilla (Espagne) : Díada.

Haines, A., Kovats, R. S., Campbell-Lendum, D. et Corvalan, C. (2006). Climate change and human health : impacts, vulnerability, and mitigation. The Lancet, 367(9528), 2101-2109.

Industry Canada (2014). Digital Canada 150. Ottawa : Gouvernement du Canada.

Internet World Statistics. http://www.internetworldstats.com/stats.htm

Jenson, B. et Schnack, K. (1997). The action competence approach in environmental education. Environmental Education Research, 3(2), 163-178.

Kane, K., Chiru, C., et Ciuchete, S. G. (2012). Exploring the Eco-Attitudes and Buying Behaviour of Facebook Users. Amfiteatru Economic, 14(31), 157-171.

Kollmus, A. et Agyeman, J. (2002). Mind the gap : why do people act environmentally and what are the barriers to pro-environmental behaviour ? Environmental Education Research, 8(3), 239-260.

Le Bourdais, C., Desrosiers, H. et Gaulin, B. (1991). Étude de la dynamique familiale : problèmes de définition des concepts et des outils de mesure Institut national de la recherche scientifique -

Urbanisation, Montréal

Léger, M. T. et Freiman, V. (2016). A Narrative Approach to Understanding the Development and Retention of Digital Skills Over Time in Former Middle School Students, a Decade After Having Used One-to-One Laptop Computers. Journal of Research on Technology in Education, 48(1).

Léger, M. T. et Freiman, V. (2015). Perceptions of information and communications technology skills in middle school students using individual laptops : Toward a longitudinal perspective. Proceedings of the American Educational research Association (AERA) annual meeting. Chicago, IL.

Léger, M. T., Kerry, J., Pruneau, D. et Langis, J. (2014). Planifier pour un avenir écodurable : développer la compétence de planification écodurable dans une classe de 6e année. Canadian Journal of Environmental Education, 19, 127-144.

Léger, M. T. et Pruneau, D. (2014). L'adoption de comportements durables dans la famille : Perspectives théoriques. Éducation relative à l'environnement : Regards, recherches, réflexions, 12, 153-168.

Léger, M. T. et Pruneau, D. (2013). Challenging families to live more sustainably : A multicase study of the processes involved in adopting eco-sustainable habits in the context of family. Journal of Sustainability Education, Spring, Part II. Récupéré de : www.jsedimensions.org

Léger, M. T. et Pruneau, D. (2012). Changing family habits : a study into the process of adopting climate change mitigation behaviours. International Electronic Journal of Environmental Education, 2(2), 77-87. 
Léger, M. T. et Pruneau, D. (2011). A grounded theory perspective on eco-sustainable change in families. Ecopsychology, 3(4), 237-247.

Maiteny, P. T. (2002). Mind the gap : summary of research exploring "inner" influences on prosustainability learning and behaviour. Environmental Education Research, 8(3), 299-306.

Marleau, M.-È. (2009). Des liens à tisser entre la prise de conscience et l'action environnementales. Éducation et francophonie, 37(2), 11-32.

Neilson, J. et Stanfors, M. (2014). It's About Time ! Gender, Parenthood, and Household Divisions of Labor Under Different Welfare Regimes. Journal of Family Issues, 35(8).

Organization for Economic Co-operation and Development. (2008). OECD Household Survey on Environmental Attitudes and Behaviours : Data Corroboration. OECD Publishing. www.oecd.org/env/ consumption-innovation/44101274.pdf

Paillé, P. et Mucchielli, A. (2012). L'analyse qualitative en sciences humaines et sociales. Paris : Armand Colin.

Payne, P. (2010). Moral spaces, the struggle for an intergenerational environmental ethics and the social ecology of families : An other form of environmental education. Environmental Education Research, 16(2), 209-231.

Payne, P. (2005). Growing up green ; the household as oikos. Journal of the Home Economics Institute of Australia, 12, 2-12.

Prochaska, J. O., DiClemente, C. C. et Norcross, J. C. (1992). In search of how people change : Applications to addictive behaviours. American Psychologist, 47(9), 1102-1114.

Pruneau, D., Kerry, J. et Langis, J. (2013). Étude des compétences propices au soin et à la transformation de l'environnement, Vertigo, 13(1).

Queensland Youth Environmental Council (2009). Youth and Environment Survey: A report on the environmental attitudes, knowledge and practices of 12 to 24 year olds. www.qyec.org.au/survey

Sauvé, L. (1997). Pour une éducation relative à l'environnement : Éléments de design pédagogique. Montréal : Guérin.

Social Sciences and Humanities Research Council (2010). Knowledge Synthesis Grants on Digital Economy. Récupéré de : www.sshrc-crsh.gc.ca/society-societe/outcomes-resultants/ knowledge_synthesis-subventions_de_synthese-eng.aspx Stake, R. (1995). The art of case study research. Thousand Oaks (CA) : Sage.

Statistics Canada. (2016). Dictionary, Census of Population. http://www12.statcan.gc.ca/censusrecensement/2016/ref/dict/fam004-eng.cfm

Sweeney, J. C., Webb, D., Mazzarol, T., et Soutar, G. N. (2014). Self-Determination Theory and Word of Mouth about Energy-Saving Behaviors : An Online Experiment. Psychology \& Marketing, 31(9), 698-716.

Whitmarsh, L. et O'Neill, S. (2011). Introduction : Opportunities for and barriers to engaging individuals with climate change. In L. Whitmarsh, S. O'Neill et I. Lorenzoni (Eds.) (p, 1-14). Engaging the public with climate change : behaviour change and communication. Washington : Earthscan.

Widmer, E., Kellerhals, J. et Levy, R. (2004). Types of Conjugal Networks, Conjugal Conflict and Conjugal Quality. European Sociological Review, 20(1), 63-77. 


\section{RÉSUMÉS}

L'intégration de modes de vie écoresponsables représente un défi difficile à surmonter pour grand nombre de Canadiens. Cette étude qualitative vise à explorer l'adoption collective d'actions écologiques dans la famille, un contexte de transformation socioécologique peu examiné en recherche. Tenant compte de l'utilisation populaire des médias sociaux aujourd'hui, ce projet cherche à mieux comprendre comment une famille peut harmoniser davantage ses rapports avec l'environnement à l'aide du numérique. Pour certains auteurs, les médias sociaux numériques peuvent, en effet, influencer le choix de se comporter de façon plus écologique en exerçant une pression sociale positive pour l'action écologique. Il semble que ces outils numériques peuvent en particulier contribuer à réduire la consommation en énergie à la maison. Dans notre projet, il s'agit de voir comment Facebook peut contribuer au développement d'une écocitoyenneté familiale en permettant à des familles voulant adopter des modes de vie plus écologiques, de s'entre-aider comme communauté d'action. L'analyse descriptive d'une participation quotidienne au sein d'un groupe privé Facebook et l'analyse thématique d'entretiens d'explicitation après quatre mois de participation montrent que les actions environnementales mises en place par les familles impliquées semblent avoir persisté du début à la fin du projet. Bien que ces actions étaient relativement simples, nos résultats semblent indiquer que la participation à un réseau social virtuel peut contribuer à un plus haut niveau de conscience écologique et d'engagement envers l'environnement dans la famille, surtout chez les enfants des familles participant à ce projet.

Adopting eco-responsible habits represents a difficult challenge for a large number of Canadiens. This qualitative study explores the adoption of collective ecological actions in families, a context of socioecological transformation rarely looked at in the scientific literature. Given the popularity of social media today, the present study looks to further understand how a typical family can establish a better relationship with the environment with the help of technology. For some authors, online social media can, in fact, influence one's choice to act more ecologically likely through positive social pressure. It seems such digital tools can lead to reductions in household energy use. Our study attempts to find out how Facebook can contribute to the development of stronger ecological habits in families who want to heighten their level of ecocitizenship by collaborating with other likeminded families. We present a descriptive analysis of daily participation within the dedicated Facebook group as well as a thematic qualitative analysis of exit interviews, after four month of project involvement, which both support the finding that environmental actions adopted at the beginning of the project persisted through the entire participation period. Though these actions were still relatively simple, our results point to a higher level of environmental awareness and engagement among families who participated in the project's Facebook group, especially in children.

\section{INDEX}

Mots-clés : engagement environnemental, écocitoyenneté, étude de cas

Keywords : environmental engagement, eco-citizenship, case study 


\section{AUTEURS}

\section{MICHEL T. LÉGER}

Michel T. Léger est professeur agrégé spécialisé en éducation relative à l'environnement (ERE) à l'Université de Moncton, où il enseigne des cours en éducation environnementale, en planification pédagogique et en didactique des sciences. Ses recherches portent principalement sur la place des technologies en éducation relative à l'environnement et sur le développement d'actions environnementales en contexte de famille. Il supervise régulièrement des étudiantes et étudiants aux cycles supérieurs, en éducation ainsi qu'au programme de la Maitrise en études de l'environnement. Avant de devenir professeur d'université, il a enseigné les sciences à l'école secondaire pendant 11 ans au District scolaire francophone Sud, au Nouveau-Brunswick.

\section{SHAWN MARTIN}

Shawn Martin a diplômé du programme de la Maitrise en études de l'environnement à l'Université de Moncton et enseigne maintenant au district scolaire francophone Sud, au Nouveau-Brunswick. 